3. - Perturbation of normal operators, Proc. Amer. Math. Soc. vol. 5 (1954) pp. $103-110$.

4. F. Rellich, Störungstheorie der Spectralzerlegung, III, Math. Ann. vol. 116 (1939) pp. 555-570; V, Math. Ann. vol. 118 (1942) pp. 462-484.

5. B. v. Sz.-Nagy, Perturbations des transformations lineaires fermées, Acta Univ. Szeged. vol. 14 (1951) pp. 125-137.

6. F. Riesz and B. v. Sz.-Nagy, Leçons d'analyse fonctionnelle, Budapest, 1953.

7. H. Wielandt, Pairs of normal matrices with property L, Journal of Research of the National Bureau of Standards vol. 51 (1953) pp. 89-90.

8. F. Wolf, Analytic perturbation of operators in Banach spaces, Math. Ann. vol. 124 (1952) pp. 317-333.

University of California, Berkeley

UNIVERSITY OF WASHINGTON

\title{
ON A THEOREM OF MAGNUS
}

\section{EDWIN HEWITT AND EUGENE P. WIGNER ${ }^{1}$}

1. In a recent paper $[2],{ }^{2} \mathrm{~W}$. Magnus has shown that analogues of the Fourier inversion and Plancherel theorems hold for matrixvalued functions on the real line $R$. We propose to show that these theorems actually hold for an arbitrary locally compact Abelian group, and that Magnus's inversion integral (1. c. (1.4)) can be simplified. For all group- and integral-theoretic notation, terms, and facts used here without explanation, see [1].

2. Let $G$ be a locally compact Abelian group, written additively, with character group $X$. Elements of $G$ will be denoted " $s$ ", " $t$ ", and elements of $X$ by " $\chi$ ", with or without subscripts. The differential of Haar measure on $G[X]$ will be denoted $d t[d \chi]$ and these measures are to be so chosen that equality obtains in the Fourier inversion theorem [1, p. 143] and Plancherel's theorem [1, p. 145].

2.1. Let $U$ be a continuous $n$-dimensional unitary representation of $G$, so that: $U(s+t)=U(s) U(t)$ for all $s, t \in G ; U(0)=I$; and all coefficients $u_{j k}$ of $U$ are continuous functions on $G$. Then the reduction theorem states that there exist a unitary matrix $V$ and characters $\chi_{1}, \cdots, \chi_{n} \in X$ such that

Received by the editors February 16, 1956 and, in revised form, October 1, 1956.

1 The first-named author is a fellow of the John Simon Guggenheim Memorial Foundation.

2 Numbers in brackets refer to the bibliography. 


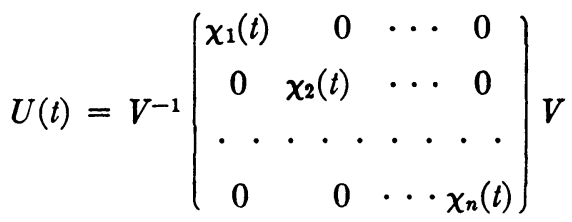

for all $t \in G$.

The set $\left\{\chi_{1}, \cdots, \chi_{n}\right\}$ of characters is completely determined by $U$, although obviously their order is not.

2.2. We now select a symmetric compact neighborhood $A$ of the identity in $X$ having (finite) positive measure. Let $E=E\left(\chi_{1}, \cdots, \chi_{n}\right)$ be the function on $X^{n}$ that is equal to 1 if $\chi_{j} \chi_{k}^{-1} \in A$ for all $j, k$ and is equal to 0 otherwise. It is obvious that

$$
E\left(\chi_{1}, \cdots, \chi_{n}\right)=E\left(\chi_{1} \chi, \cdots, \chi_{n} \chi\right)
$$

for all $\chi \in X$.

3. Let $f \in L_{1}(G)$. We say that Fourier inversion holds for $f$ if $\hat{f} \in L_{1}(X)$ and $\int_{X} \hat{f}(\chi) \overline{\chi(s)} d \chi=f(s)$ for all $s \in G$. (Recall that $\hat{f}(\chi)$ $=\int_{G} f(t) \chi(t) d t$. $)$ It is known $[1$, p. 143] that Fourier inversion holds if $f$ is in $L_{1}(G)$ and is also a linear combination of continuous positive definite functions. We can now establish our generalization of Magnus's theorem.

3.1. TheOREM. Let $F=F(t)$ be an $n \times n$ complex matrix function on $G$ such that $F_{j k} \in L_{1}(G)$ and Fourier inversion holds for $F_{j k}$, for all coefficients $F_{j k}$ of $F$. For every representation as in 2.1 , let

$$
F^{\wedge}(U)=\int_{G} F(t) U(t) d t .
$$

Then for all $s \in G$, the equality

$$
\kappa \cdot F(s)=\int_{X^{n}} F^{\frown}(U) U(-s) E\left(\chi_{1}, \cdots, \chi_{n}\right) d \chi_{1} \cdots d \chi_{n}
$$

holds, where $\kappa$ depends only on $n$ and $A$ and $0<\kappa<\infty$. The integral in 3.1 .2 is an $n$-fold group integral over $X^{n}$, in which the integrand vanishes unless $\chi_{j} \chi_{k}^{-1} \in A$ for all $j$ and $k$. The matrix $F^{-}(U) U(-s)$ depends symmetrically on the characters $\chi_{1}, \cdots, \chi_{n}$ associated with $U$ by 2.1.1, and hence the integrand in 3.1.2 is well defined.

Proof. Let $j$ and $k$ be arbitrary integers, $1 \leqq j \leqq n$ and $1 \leqq k \leqq n$. Using 3.1.1 and 2.1.1, we can write the $(j, k)$ th entry of the integral in 3.1 .2 as 
3.1.3 $\sum_{\alpha, \beta=1}^{n} \int_{X^{n}} \int_{G} F_{j \alpha}(t) \chi_{\beta}(t-s) \bar{v}_{\beta \alpha} v_{\beta k} E\left(\chi_{1}, \cdots, \chi_{n}\right) d t d \chi_{1} \cdots d \chi_{n}$.

(Here and also below we make free use of Fubini's theorem.) For each $\alpha$ and $\beta$, the integral in 3.1 .3 can be written as

$3.1 .4 \quad \bar{v}_{\beta \alpha} v_{\beta k} \int_{X^{n}} \hat{F}_{j \alpha}\left(\chi_{\beta}\right) \chi_{\beta}(-s) E\left(\chi_{1}, \cdots, \chi_{n}\right) d \chi_{1} \cdots d \chi_{n}$.

To evaluate 3.1.4, suppose for simplicity that $\beta=1$. The integral in 3.1.4, taken over $\chi_{2}, \cdots, \chi_{n}$, will be independent of the value of $\chi_{1}$. To see this, we substitute 2.2 .1 in 3.1 .4 and obtain

$$
\begin{array}{rl}
\int_{X^{n}} & E\left(\chi_{1}, \chi_{2}, \cdots, \chi_{n}\right) d \chi_{2} \cdots d \chi_{n} \\
= & \int_{X^{n}} E\left(\chi_{1} \chi, \chi_{2} \chi, \cdots, \chi_{n} \chi\right) d \chi_{2} \cdots d \chi_{n} \\
= & \int_{X^{n}} E\left(\chi_{1} \chi, \chi_{2}, \cdots, \chi_{n}\right) d \chi_{2} \cdots d \chi_{n} .
\end{array}
$$

The last step follows because the Haar integral over $X$ is invariant. Since $\chi$ is arbitrary in $X$, our assertion about 3.1.4 follows. By hypothesis, Fourier inversion holds for $F_{j \alpha}$. Therefore, denoting the value of 3.1 .5 by $\kappa$ (it is easy to see that $0<\kappa<\infty$ ), we can write 3.1.4 as

3.1 .6

$$
\bar{v}_{\beta \alpha} v_{\beta k} \kappa F_{j \alpha}(s) .
$$

Hence 3.1 .3 is equal to $\sum_{\alpha, \beta=1}^{n} \bar{v}_{\beta \alpha} v_{\beta k} \kappa F_{j \alpha}(s)=\sum_{\alpha=1}^{n} \delta_{\alpha k} \kappa F_{j \alpha}(s)$ $=\kappa F_{j k}(s)$. This proves the theorem.

3.2. REMARK. The foregoing proof shows that the matrices $V$ and $V^{-1}$ in 2.1.1 play a quite inessential rôle for Fourier inversion. They do not affect the value of the integral 3.1.2, although $F^{\wedge}(U) U(-s)$ does depend upon the choice of $V$. This fact makes it unnecessary to integrate over the unitary group in order to invert the mapping $F \rightarrow F^{-}$. Note that Magnus's inversion formula involves integrating over the unitary group.

4. The Plancherel theorem is very easy to establish in the present context. For a matrix $M$, let $M^{*}$ be the conjugate transpose of $M$ and let $\operatorname{Tr} M$ be the trace of $M$.

4.1. TheOREM. Let $F=F(t)$ be an $n \times n$ complex matrix-valued function on $G$ such that $F_{j k} \in L_{1}(G) \cap L_{2}(G)$ for all coefficients $F_{j k}$ of $F$. Let $F^{-}$(U) be defined as in 3.1.1. Then 
4.1 .1

$$
\kappa \operatorname{Tr} \int_{G} F(t) F^{*}(t) d t
$$

$$
=\operatorname{Tr} \int_{X^{n}} F^{\frown}(U) F^{\frown *}(U) E\left(\chi_{1}, \cdots, \chi_{n}\right) d \chi_{1} \cdots d \chi_{n} .
$$

Proof. Let $D\left(\chi_{1}, \cdots, \chi_{n}\right)$ denote the diagonal matrix with entries $\chi_{1}, \cdots, \chi_{n}$. For a fixed representation $U$, write $U=V^{-1} D\left(\chi_{1}, \cdots\right.$, $\left.\chi_{n}\right) V$. Let $P(t)=V F(t) V^{-1}$, and let $Q\left(\chi_{1}, \cdots, \chi_{n}\right)$ be the matrix $\left\{\hat{P}_{j k}\left(\chi_{k}\right)\right\}_{j, k=1}^{n}$. Then we have

4.1 .2

$$
F^{\frown}(U)=\int_{G} F(t) U(t) d t=V^{-1} \cdot \int_{G} P(t) \cdot D\left(\chi_{1}(t), \cdots, \chi_{n}(t)\right) d t \cdot V
$$

$$
=V^{-1} Q\left(\chi_{1}, \cdots, \chi_{n}\right) V .
$$

It is plain from 4.1.2 that

$$
\operatorname{Tr}\left(Q Q^{*}\right)=\operatorname{Tr}\left(F^{\frown} F^{\wedge}\right) .
$$

Hence we find

$$
\operatorname{Tr}=\int_{X^{n}} F^{\frown} F^{\frown *}(U) E\left(\chi_{1}, \cdots, \chi_{n}\right) d \chi_{1} \cdots d \chi_{n}
$$

4.1.3 $=\operatorname{Tr} \int_{X^{n}} Q\left(\chi_{1}, \cdots \chi_{n}\right) Q^{*}\left(\chi_{1}, \cdots \chi_{n}\right) E\left(\chi_{1}, \cdots, \chi_{n}\right) d \chi_{1} \cdots d \chi_{n}$

$$
=\sum_{j, k=1}^{n} \int_{X^{n}}\left|\hat{P}_{j k}\left(\chi_{k}\right)\right|^{2} E\left(\chi_{1}, \cdots, \chi_{n}\right) d \chi_{1} \cdots d \chi_{n}
$$

By the usual Plancherel theorem and the argument used in 3.1, we can rewrite the last term in 4.1 .3 as

4.1 .4

$$
\kappa \cdot \sum_{j, k=1}^{n} \int_{G}\left|P_{j k}(t)\right|^{2} d t .
$$

Now using again elementary facts about the trace, we can rewrite 4.1.4 as

4.1 .5

$$
\kappa \int_{G} \operatorname{Tr}\left[P(t) P^{*}(t)\right] d t=\kappa \int_{G} \operatorname{Tr}\left[V F(t) F^{*}(t) V^{-1}\right] d t
$$

$$
=\kappa \int_{G} \operatorname{Tr}\left[F(t) F^{*}(t)\right] d t=\kappa \operatorname{Tr} \int_{G} F(t) F^{*}(t) d t .
$$

Combining 4.1.2-4.1.5, we have the present theorem.

4.2. REMARK. If some coefficient $F_{j k}$ of $F$ is in $L_{2}(G)$ but not in 
$L_{1}(G)$, then the integral 3.1.1 does not exist as an absolutely convergent integral. Nevertheless, a theorem analogous to 4.1 follows with equal ease.

5. We note finally the obvious fact that an "isomorphic" theory can be constructed using the integral

$$
\widehat{F}(U)=\int_{G} U(t) F(t) d t
$$

and the inversion integral

$$
\int_{X^{n}} U(-s)^{\frown} F(U) E\left(\chi_{1}, \cdots, \chi_{n}\right) d \chi_{1} \cdots d \chi_{n}
$$

\section{BIBLIOGRAPHY}

1. Lynn H. Loomis, An introduction to abstract harmonic analysis, New York, Van Nostrand, 1953.

2. Wilhelm Magnus, $A$ Fourier theorem for matrices, Proc. Amer. Math. Soc. vol. 6 (1955) pp. 880-890. (Also appeared as Division of Electromagnetic Research, Institute of Mathematical Sciences, New York University, Research Report No. BR-8 (1954)).

Institute for Advanced Study, UNIVERSITY OF WASHINGTON AND Princeton University 